\title{
Modeling the crossover variability in a differentially rotating star
}

\author{
V. R. Khalack ${ }^{1,2}$ \\ 1 Main Astronomical Observatory, 27 Zabolotnogo Str., 03650 Kyiv, Ukraine \\ e-mail: khalack@mao.kiev .ua \\ 2 Isaac Newton Institute of Chile, Kiev Branch
}

Received 12 October 2001 / Accepted 8 January 2002

\begin{abstract}
Reconstruction of phase-dependent crossover effect variations is performed for a differentially rotating star assuming that point-like magnetic charges are distrubuted under the stellar surface. Models adopting the solar differential rotation law and an exponential rotation law are considered and compared to the case of a rigidly rotating star. It is shown that neglect of differential stellar rotation leads to underestimation of the equatorial rotational velocity by $\sim 2-5 \%$.
\end{abstract}

Key words. stars: magnetic fields - stars: rotation - Sun: rotation

\section{Introduction}

Variations of the surface magnetic field with stellar rotation are observed for the majority of chemically peculiar (CP) stars (Romanyuk 1997). These variations are thought to be caused by nonuniformity of the magnetic fields on the surface of CP stars. Probably, the location of patches of chemical elements (spots or rings) on the stellar surface is related to the distribution of the magnetic fields (Shavrina et al. 2001). Therefore, it is evident that precise reconstruction of the surface magnetic field can help provide a comprehensive element abundance analysis and anomaly mapping.

The last two decades have seen rapid progress in the measurement of the surface magnetic fields (Mathys 1988, 1991, 1995a, 1995b; Mathys \& Hubrig 1997; Leone \& Catanzaro 2001). First introduced by Mathys (1988), the moment technique relates the line profile asymmetries (while measuring the Stokes $V$ parameter) to the mean values of the longitudinal magnetic field (first moment) and the crossover effect (second moment) for a given phase of stellar rotation.

Special attention should be paid to the crossover effect, since it reflects the result of a joint action of the Zeeman and Doppler effects on the investigated line profile of the Stokes $V$ parameter (Mathys 1995a). Such an effect was first detected in HD 125248 and was named a crossover effect by Babcock (1951). This term comes from the fact that the effect usually achieves its maximum value when the mean longitudinal magnetic field reverses its sign, i.e. it crosses zero from one polarity to another. For example, in the oblique rotator model (when the magnetic and rotational axes do not coincide) with a dipolar magnetic field for a rigidly rotating star (Stibbs 1950), the longitudinal component of the local magnetic field is different in the two halves of the stellar disk at the surface points with equal radial velocity of rotation. Apparently, this difference is maximal at the particular moment when the two magnetic poles approach the stellar limb, while the mean longitudinal magnetic field is close to zero.

Recently, Mathys (1995a) proposed a new term, asymmetry of the longitudinal field, emphasizing the relationship of the cross-over to the asymmetry in the distribution of the longitudinal component of the magnetic field, when considering the two halves of the visible stellar disk separated by the plane including the line of sight and the stellar rotational axis. In general, for a differentially rotating star with a complicated structure of its surface magnetic field it is not evident that the maximum of the effect coincides with the moment of sign reversion of the mean longitudinal magnetic field. Nevertheless, Babcock's term crossover effect is usually used to refer to the value that is actually derived from measurements of the second-order moment of the $V$ line profile (Mathys 1995a).

\section{Model}

As mentioned above, the observable value of the crossover effect depends on the combined contribution of the Zeeman and Doppler effects to the line profile asymmetry of the Stokes $V$ parameter averaged over the visible stellar disk for a given rotation phase. Therefore, at first it is necessary to model the structure of the surface magnetic field together with the velocity law for stellar rotation.

Among the known models the most appropriate one is the oblique rotator model proposed by Gerth et al. (1997, 1998), especially its modification (Khalack et al. 2001) 


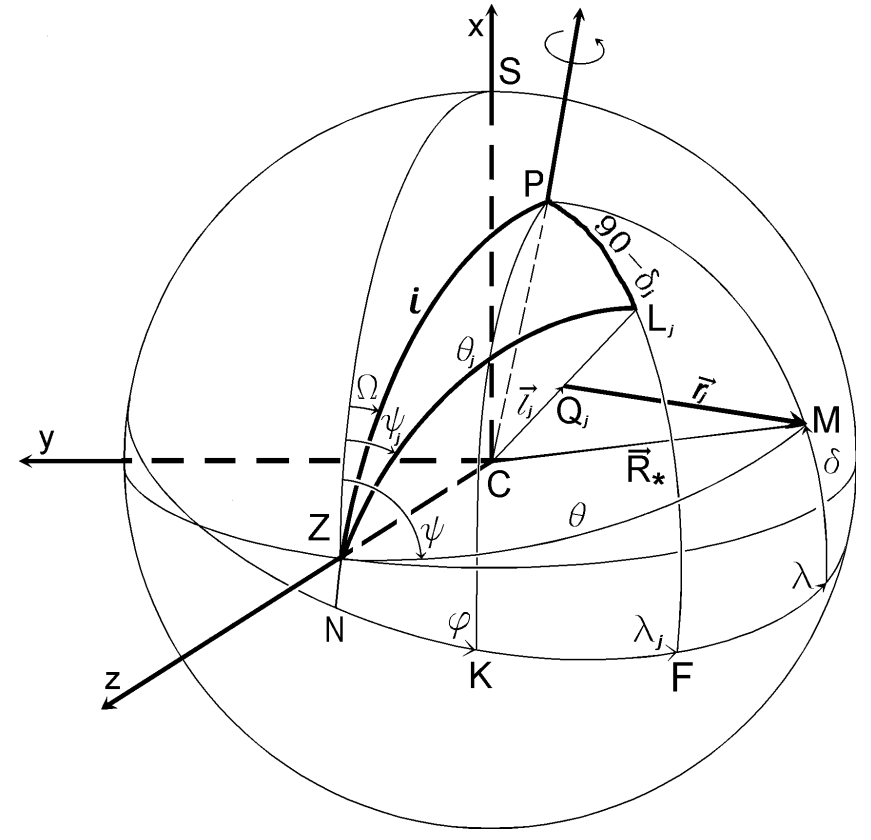

Fig. 1. Geometry of the oblique rotator model with one pointlike magnetic field source.

that allows one to reconstruct with high accuracy the geometry of the surface magnetic field under the given distribution of the point field sources embedded in the stellar body. In order to simplify the calculations, let us consider the case of centered symmetric magnetic dipole with two magnetic charges located close to the stellar centre. Then the modeled mean longitudinal magnetic field (directed along Z-axis in Fig. 1) varies with phase of the stellar rotation in the following way (Khalack et al. 2001):

$B_{z} \simeq \frac{2 a Q}{R_{\star}^{2}}(3 \cos \theta[A \sin \theta+Z \cos \theta]-Z)$,

where $A=X \cos (\psi-\Omega)+Y \sin (\psi-\Omega)$ and

$\left\{\begin{array}{l}X=\cos \beta \sin i-\sin \beta \cos i \cos (\varphi+\lambda) ; \\ Y=\sin \beta \sin (\varphi+\lambda) ; \\ Z=\cos \beta \cos i+\sin \beta \sin i \cos (\varphi+\lambda) .\end{array}\right.$

Here $\theta$ and $\psi$ specify an arbitrary point $\mathbf{M}$ on the stellar surface in the spherical reference frame related to the observer. The variables $\delta=90^{\circ}-\beta$ and $\lambda$ determine orientation of the dipolar magnetic axis (defined by location of the magnetic charges) in the spherical frame related to the star (see Fig. 1). Angles $i$ and $\Omega$ define the inclination of the rotational axis to the line of sight and its plane-ofsky orientation relative to the north celestial pole, respectively. The variables $Q$ and $a \ll 1$ determine the modulus of the magnetic charge and its distance from the centre of the star, expressed in the units of stellar radius $R_{\star}$. From the spherical triangle $\mathbf{Z} \mathbf{P} \mathbf{L}_{j}$ with the known angles $\Omega$ and $i$, the relation between the two mentioned spherical frames is derived as (omitting the subscript $j$ ):

$\left\{\begin{array}{l}\cos \theta=\sin \delta \cos i+\cos \delta \sin i \cos (\varphi+\lambda), \\ \sin \theta=\sqrt{1-\cos ^{2} \theta}\end{array}\right.$ and

$$
\left\{\begin{array}{l}
\cos (\psi-\Omega)=\frac{\sin \delta-\cos i \cos \theta}{\sin \theta \sin i}, \\
\sin (\psi-\Omega)=\cos \delta \frac{\sin (\varphi+\lambda)}{\sin \theta} .
\end{array}\right.
$$

\section{Crossover effect for a differentially rotating star}

\subsection{The solar differential rotation law}

Stars are expected to rotate differentially when energy transfer to the surface is dominated by convection. Let us consider a spherically symmetric star with a decrease in the angular rotation rate from equator to pole. Our Sun (if we neglect its oblation) has the differential rotation law of the form (Gadun et al. 1985) $\tilde{v}(\delta)=v_{\mathrm{e}}^{\mathrm{d}} \cos \delta\left(1-b \sin ^{2} \delta\right)$, where $\delta$ is the latitude, $v_{\mathrm{e}}^{\mathrm{d}}$ specifies the equatorial velocity and parameter $0<b \leq 1$ (the case of $b=0$ corresponds to a rigidly rotating star). Here we neglect the contribution of turbulence and meridional circulation to the surface velocity at a given latitude. Correspondingly, the line-of-sight projection of the velocity vector at a surface point $\mathbf{M}(\delta, \lambda)$ is

$\tilde{v}_{z}=v_{\mathrm{e}}^{\mathrm{d}} \cos \delta\left(1-b \sin ^{2} \delta\right) \sin i \sin (\varphi+\lambda)$.

In the spherical frame related to an observer this projection can be expressed as (see Fig. 1 and Eqs. (3), (4)):

$$
\begin{aligned}
\tilde{v}_{z}= & v_{\mathrm{e}}^{\mathrm{d}} \sin i \sin (\psi-\Omega) \sin \theta \times \\
& {\left[1-b(\cos \theta \cos i+\sin \theta \sin i \cos (\psi-\Omega))^{2}\right] . }
\end{aligned}
$$

As follows from the definition of the crossover effect (Mathys 1995a), contribution from an arbitrary surface point $\mathbf{M}(\theta, \psi)$ is proportional to $B_{\mathrm{z}}(1)$ due to the Zeeman effect, and to $\tilde{v}_{\mathrm{z}}(6)$ due to the Doppler effect: $B_{\mathrm{c}} \simeq B_{\mathrm{z}} \cdot v_{\mathrm{z}}$. Adopting a limb darkening law for the star in the form $1-u+u \cos \theta$ (where $0 \leq u \leq 1$ ) and averaging $B_{\mathrm{c}}$ over the visible stellar hemisphere in accordance with Landolfi et al. (1998), one can obtain the following theoretical expression for the observed value of the crossover effect:

$$
\begin{aligned}
\left\langle B_{\mathrm{c}}\right\rangle^{\mathrm{d}} \simeq & v_{\mathrm{e}}^{\mathrm{d}} c_{0} \frac{4 a Q}{R_{\star}^{2}}(Y \sin i) \frac{1}{2240}\{448-168 u- \\
& \left.b\left[64-29 u+\cos ^{2} i(128-23 u)\right]\right\}
\end{aligned}
$$

where $c_{0}=3 /(3-u)$ and $Y$ is defined in (2). For the rigidly rotating star (case $b=0$ ) it transforms into

$$
\left\langle B_{\mathrm{c}}\right\rangle^{\mathrm{r}} \simeq v_{\mathrm{e}}^{\mathrm{r}} c_{0} \frac{4 a Q}{R_{\star}^{2}}(Y \sin i) \frac{8-3 u}{40},
$$

where $v_{\mathrm{e}}^{\mathrm{r}}$ specifies the equatorial velocity. This expression is in good agreement with the previously obtained result (Bagnulo et al. 1996) for the model of an oblique rotator with the centered symmetric magnetic dipole, where the factor $4 a Q / R_{\star}^{2}$ is proportional to the field magnitude $B_{\mathrm{d}}$ at the magnetic pole.

In order to simplify the analysis of the obtained results it is useful to introduce the function $f(b, u, i)$ defined as 


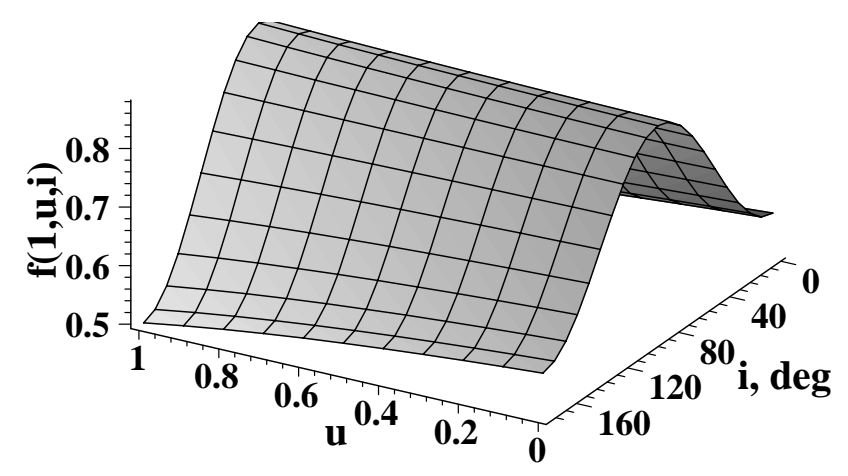

Fig. 2. Shape of the function $f(b=1, u, i)$ defined by the Eq. (10) for the solar differential rotation law.

ratio of the crossover magnitude in the model of rigidly rotating star to the crossover magnitude in the differentially rotating model:

$$
\frac{\left\langle B_{\mathrm{c}}\right\rangle^{\mathrm{r}}}{\left\langle B_{\mathrm{c}}\right\rangle^{\mathrm{d}}} \simeq \frac{v_{\mathrm{e}}^{\mathrm{r}}}{v_{\mathrm{e}}^{\mathrm{d}}} \frac{1}{f(b, u, i)},
$$

where (see Eqs. (7), (8))

$$
f(b, u, i)=1-b \frac{64-29 u+(128-23 u) \cos ^{2} i}{56(8-3 u)} .
$$

It is obvious that $f(b, u, i)<1$ for an arbitrary value of the angle $i, 0 \leq u<1$ and $0<b \leq 1$ (see, for example, Fig. 2). To compare two models with different rotation laws let us postulate $\left\langle B_{\mathrm{c}}\right\rangle^{\mathrm{r}} /\left\langle B_{\mathrm{c}}\right\rangle^{\mathrm{d}}=1$, since the same observations of the crossover effect are employed. Correspondingly, ratio of the equatorial velocities given by these two models is (see Eq. (9))

$$
\frac{v_{\mathrm{e}}^{\mathrm{r}}}{v_{\mathrm{e}}^{\mathrm{d}}} \simeq f(b, u, i)<1 .
$$

Thus, adoption of rigid rotation in modeling of the crossover effect leads to underestimation of the real equatorial velocity of differentially rotating star.

The plot of function $f(b=1, u, i$ ) (see Eq. (10)) is given in Fig. 2. As follows from this figure, maximal underestimation (that corresponds to the minimum of function $f(b, u, i)$ ) of the real equatorial velocity in the model of a rigidly rotating star is about $50 \%$ of the velocity estimate for $b=1, u \approx 1$ and $i=0^{\circ}$ or $180^{\circ}$. For the main sequence stars with $T_{\text {eff }}=6000{ }^{\circ} \mathrm{K} \div 30000{ }^{\circ} \mathrm{K}$ and $\log g=4.0 \div 5.0$ (spectral type B, A and F) the limb darkening coefficient $u$ is about $0.3 \div 0.7$ (Diaz-Cordoves et al. 1995). Accepting the mean inclination of the rotational axis to the line of sight to be $i=45^{\circ}$ (Landstreet \& Mathys 2000) and assuming $b=0.1 \div 0.2$, as for the case of the Sun (Gadun et al. 1985), Eq. (10) leads to underestimation of the order of $2-5 \%$ (see. Fig. 4) for the real equatorial velocity of the differentially rotating star. It is of the order of the typical error bar $\sigma\left[v_{\mathrm{e}}^{\mathrm{r}}\right]$ in modeling estimates of the rotational velocity on the stellar equator.

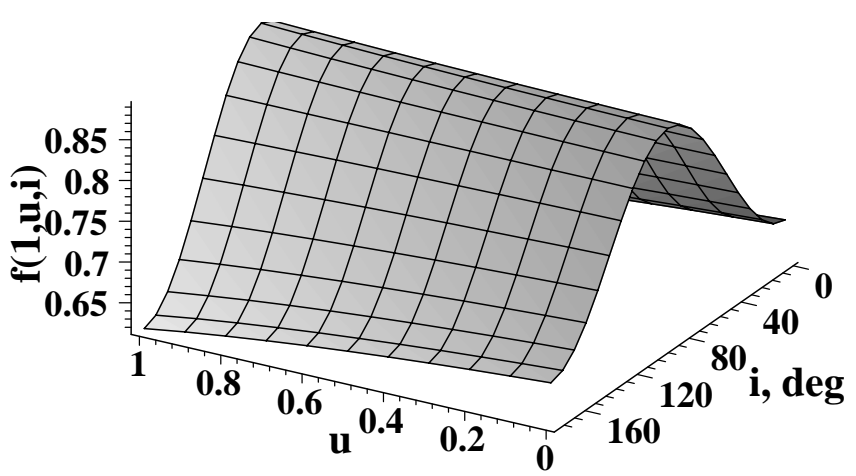

Fig. 3. The same as Fig. 2, but for the differential rotation with an exponential law $(14,15)$.

\subsection{Differential rotation with an exponential law}

In the stability analysis of a magnetized, differentially rotating star researchers frequently use an exponential law (Uprin 1996) that significantly simplifies the analytical calculations. In such a case the rotational velocity depends on the stellar latitude $\delta$ as $\tilde{v}(\delta)=v_{\mathrm{e}}^{\mathrm{d}} \cos \delta \exp \left(-b \cos ^{2} \delta\right)$, where $v_{\mathrm{e}}^{\mathrm{d}}$ specifies the equatorial velocity, and the parameter $b$ is restricted as $0<b \leq 1$ (the case of $b=0$ corresponds to a rigidly rotating star). At an arbitrary surface point $\mathbf{M}(\delta, \lambda)$, projection of the rotational velocity onto the line of sight is (see Fig. 1)

$\tilde{v}_{\mathrm{z}}=v_{\mathrm{e}}^{\mathrm{d}} \cos \delta \exp \left(-b \cos ^{2} \delta\right) \sin i \sin (\varphi+\lambda)$.

In order to average the crossover effect $B_{\mathrm{c}} \simeq B_{\mathrm{z}} \cdot \tilde{v}_{\mathrm{z}}$ one has to integrate the product of Eqs. (1) and (12) over the visible stellar hemisphere. Unfortunately, this integration cannot be done analytically. As a possible way to simplify the calculations, the exponent in the velocity expression (12) can be expanded into a series in the small parameter $b$

$$
\begin{aligned}
\tilde{v}_{\mathrm{z}}(\delta)= & v_{\mathrm{e}}^{\mathrm{d}} \cos \delta\left\{1-b \cos ^{2} \delta+\frac{b^{2}}{2} \cos ^{4} \delta-\frac{b^{3}}{6} \cos ^{6} \delta+\right. \\
& \left.\ldots+(-1)^{n} \frac{b^{n}}{n !} \cos ^{2 n} \delta\right\} \sin i \sin (\varphi+\lambda) .
\end{aligned}
$$

The resulting series has an infinite convergence radius. Therefore, it suffices to take into account only the first few terms to provide an acceptable accuracy for the final result. Performing the same calculations as in the previous subsection, one can obtain the following expression for the function analyzed:

$f(b, u, i)=1-b P_{1}+b^{2} P_{2}-b^{3} P_{3}$,

where

$$
\left\{\begin{aligned}
P_{1}= & \frac{64-29 u+(128-23 u) \cos ^{2} i}{56(8-3 u)} \\
P_{2}= & \frac{128-65 u+(512-134 u) \cos ^{2} i+63 u \cos ^{4} i}{672(8-3 u)} \\
P_{3}= & \frac{2560-1405 u+(15360-4965 u) \cos ^{2} i}{88704(8-3 u)}+ \\
& \frac{3465 u \cos ^{4} i-231 u \cos ^{6} i}{88704(8-3 u)}
\end{aligned}\right.
$$




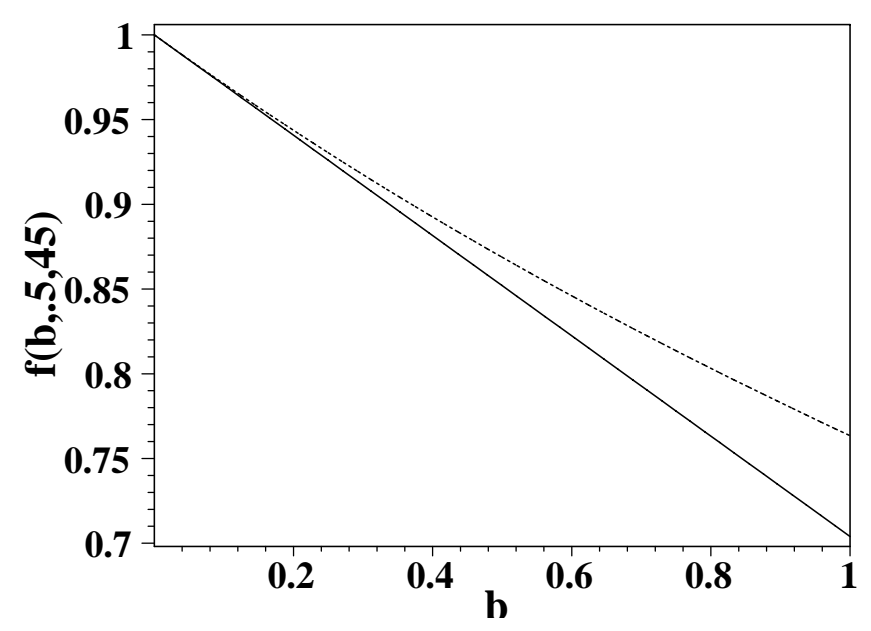

Fig. 4. Dependence of the analyzed function $f$ on the parameter $b$ in the model with the solar differential rotation law (solid line) and in the model with an exponential law (dotted line).

Only the first four terms in $b$ are taken into account in the above expression. For the minimum value of the function $f\left(\min [f(b, u, i)]=0.62\right.$ at $b=1, u \approx 1$ and $i=0^{\circ}$ or $\left.180^{\circ}\right)$, neglect of the rest of the terms leads to an error of 0.002 . The new function (14) depends mainly on $u$ and $i$ in the same manner as the function specified by Eq. (10) (see Fig. 3). On the other hand, its decrease with $b$ is comparatively slower than for the function (10) describing the solar differential rotation law (see Fig. 4). Consequently, for the star with an exponential law of differential rotation, the model of a rigidly rotating star gives a relatively smaller underestimation of errors for the equatorial velocity.

\section{Summary}

This paper is devoted to modeling of the mean crossover effect in a differentially rotating, spherically symmetric star with a centered magnetic dipole. Geometry of the surface magnetic field is derived for the point field sources with the magnetic charges embedded in the stellar body. This provides high accuracy for the field reconstruction (Khalack et al. 2001).

Since the crossover effect is defined by the joint action of the Zeeman and Doppler effects, the corresponding theoretical expression (7) for its mean (averaged over the visible stellar hemisphere) value is obtained here for the differentially rotating star with a solar-like rotation law (5). It is shown that neglect of the differential character of the stellar rotation leads to underestimation of the real equatorial velocity up to $2-5 \%$, comparable to the errors $\sigma\left[v_{\mathrm{e}}^{\mathrm{r}}\right]$ in model estimates of the stellar rotation. Comparatively smaller errors appear in the model considering the differentially rotating star with an exponential rotation law for the same value of the parameter $b$.

Nevertheless, the value (10) (related to underestimation of the equatorial velocity) is very sensitive to the parameter $b$ (see Fig. 4) that specifies the rate of decrease in the angular rotation of the star from equator to pole.
Thus, in a general case, for correct modeling of the observable variability of the values related to the surface magnetic field (the mean longitudinal magnetic field, the crossover effect, etc.), the possibility of differential rotation of the star should be comprehensively considered and then taken into account, if necessary.

Strong differential rotation characterized by sufficiently large values of $b$ would induce major distortions in the line profiles, which are not observed in the majority of CP stars (Mathys 2001). Therefore, in the case of differential rotation of these stars, the probable value of $b$ is not very high and the respective underestimation error of the real equatorial velocity is of the order of the model uncertainty due to other effects. Consequently, application of the model with a rigidly rotating star remains valid.

Acknowledgements. Author is grateful to Prof. Y. Geroyanis, Prof. G. Mathys and Dr. S. Marchenko for useful advice and fruitful discussion.

\section{References}

Babcock, H. W. 1951, ApJ, 114, 1

Bagnulo, S., Landolfi, M., \& Landi Degl'Innocenti, E. 1996, A\&A, 308, 115

Diaz-Cordoves, J., Claret, A., \& Gimenez, A. 1995, A\&AS, 110,329

Gadun, A. S., Kostyk, R. I., \& Sheminova, V. A. 1985, Kin. \& Phys. of Cel. Bodies 15, N6, 53

Gerth, E., Glagolevskij, Yu. V., \& Scholz, G. 1997, Integral representation of the surface structure of the stellar magnetic field, in Proc. of International Conference Stellar Magnetic Fields, ed. Y. V. Glagolevskij, \& I. I. Romanyuk (Special Astrophys. Observ. Press, Moscow), 67

Gerth, E., Glagolevskij, Yu. V., \& Scholz, G. 1998, Integral representation of the stellar surface structure of the magnetic field, in Proc. of the 26th Meeting and Workshop of the Europian Working Group on CP Stars, ed. P. North, A. Schnell, \& J. Žižňovský, Contr. Astron. Obs. Skalnate Pleso vol. 27, N3, 455

Khalack, V. R., Khalack, Yu. N., Shavrina, A. V., et al. 2001, AZh, 78, N7, 655

Landolfi, M., Bagnulo, S., \& Landi Degl'Innocenti, E. 1998, A\&A, 338, 111

Landstreet, J. D., \& Mathys, G. 2000, A\&A, 359, 213

Leone, F., \& Catanzaro, G. 2001, A\&A, 365, 118

Mathys, G. 1988, A\&A, 189, 179

Mathys, G. 1991, A\&AS, 89, 121

Mathys, G. 1995a, A\&A, 293, 733

Mathys, G. 1995b, A\&A, 293, 746

Mathys, G. 2001, private communication

Mathys, G., \& Hubrig, S. 1997, A\&AS, 124, 475

Romanyuk, I. I. 1997, The Zeeman effect in stellar spectra, in Proc. of International Conference Stellar Magnetic Fields, ed. Y. V. Glagolevskij, \& I. I. Romanyuk (Special Astrophys. Observ. Press, Moscow), 11

Shavrina, A. V., Polosukhina, N. S., Zverko, J., et al. 2001, AZh, 78, N10, 902

Smith, M. D. 1994, A\&A, 287, 523

Stibbs, D. W. N. 1950, MNRAS, 110, 395

Uprin, V. A. 1996, MNRAS, 280, 149 